\title{
Genetic and environmental influences on structural brain measures in twins with autism spectrum disorder
}

\author{
John P. Hegarty II $\mathbb{1}^{1} \cdot$ Luiz F. L. Pegoraro ${ }^{2}$ Laura C. Lazzeroni $\mathbb{1}^{1,3} \cdot$ Mira M. Raman $^{1} \cdot$ Joachim F. Hallmayer $^{1}$. \\ Julio C. Monterrey ${ }^{1} \cdot$ Sue C. Cleveland ${ }^{1} \cdot$ Olga N. Wolke ${ }^{4} \cdot$ Jennifer M. Phillips ${ }^{1} \cdot$ Allan L. Reiss ${ }^{1} \cdot$ Antonio Y. Hardan $^{1}$
}

Received: 29 June 2018 / Revised: 11 September 2018 / Accepted: 12 November 2018 / Published online: 18 January 2019

(c) The Author(s) 2019. This article is published with open access

\begin{abstract}
Atypical growth patterns of the brain have been previously reported in autism spectrum disorder (ASD) but these alterations are heterogeneous across individuals, which may be associated with the variable effects of genetic and environmental influences on brain development. Monozygotic (MZ) and dizygotic (DZ) twin pairs with and without ASD (aged 6-15 years) were recruited to participate in this study. T1-weighted MRIs $(n=164)$ were processed with FreeSurfer to evaluate structural brain measures. Intra-class correlations were examined within twin pairs and compared across diagnostic groups. ACE modeling was also completed. Structural brain measures, including cerebral and cerebellar gray matter (GM) and white matter (WM) volume, surface area, and cortical thickness, were primarily influenced by genetic factors in TD twins; however, mean curvature appeared to be primarily influenced by environmental factors. Similarly, genetic factors accounted for the majority of variation in brain size in twins with ASD, potentially to a larger extent regarding curvature and subcortical GM; however, there were also more environmental contributions in twins with ASD on some structural brain measures, such that cortical thickness and cerebellar WM volume were primarily influenced by environmental factors. These findings indicate potential neurobiological outcomes of the genetic and environmental risk factors that have been previously associated with ASD and, although preliminary, may help account for some of the previously outlined neurobiological heterogeneity across affected individuals. This is especially relevant regarding the role of genetic and environmental factors in the development of ASD, in which certain brain structures may be more sensitive to specific influences.
\end{abstract}

These authors contributed equally: John P. Hegarty II, Luiz F. L. Pegoraro

Supplementary information The online version of this article (https:// doi.org/10.1038/s41380-018-0330-z) contains supplementary material, which is available to authorized users.

John P. Hegarty

hegartyj@stanford.edu

1 Department of Psychiatry and Behavioral Sciences, Stanford University, 401 Quarry Road, Stanford, CA 94305, USA

2 Department of Psychiatry, University of Campinas, Cidade Universitária Zeferino Vaz, Campinas, SP 13083-970, Brazil

3 Department of Biomedical Data Science, Stanford University, 1265 Welch Road, Stanford, CA 94305, USA

4 Department of Anesthesiology, Stanford University, 300 Pasteur Drive, Stanford, CA 94305, USA

\section{Introduction}

Autism spectrum disorder (ASD) is a neurodevelopmental disorder that affects approximately 1 in 59 children in the United States [1]. ASD is characterized by cognitive/behavioral impairments in social communication (SCI) and restricted, repetitive patterns of behavior and interests (RRB) [2], with variable presentation and severity across individuals. The etiology of ASD is also variable with up to $20-25 \%$ of cases [3] arising from rare genetic abnormalities (e.g., single-gene disorders, chromosomal abnormalities, or copy number variation) but the vast majority likely stem from multifactorial genetic influences. In these cases, genetic vulnerability may interact with environmental influences [4] to alter the development of neuronal circuits [5]. As such, there have been reports of widespread neurobiological abnormalities in the brain in ASD [6]. However, these alterations are also variable. Thus, ASD is an inordinately heterogeneous disorder in terms of etiology, neurobiology, and symptomatology, which is likely 
associated with the effects of genetic and environmental interactions on different neurobiological pathways. The application of novel approaches, such as twin studies, may help to clarify the contribution of these components on brain development in ASD.

Twin studies provide an approach for estimating the influence of genetic versus environmental factors that contribute to a disorder. For instance, studies of monozygotic (MZ) and dizygotic (DZ) twin pairs suggest a high rate of genetic influence in ASD [7-10], with more recent studies indicating a potentially greater environmental contribution than previously reported [10]. The twin study design has also been applied in neurobiological investigations to assess the effects of genetic versus environmental factors that modulate neurodevelopment. Investigations of typically-developing (TD) twins suggest that brain volume (up to 90\%) [11, 12], surface area (71-89\%), and cortical thickness (69-81\%) $[13,14]$ are primarily influenced by genetic factors. This is relevant for the study of ASD because there are numerous reports of abnormal growth patterns of the brain, which indicate early overgrowth followed by a possible normalization later in life [15]. To date, the application of the twin design into neurobiological studies of ASD has been limited with most focusing on MZ twin pairs [16-18]. These studies largely corroborate reports of volumetric alterations in ASD, particularly regarding white matter (WM), and further suggest that cerebellar volume may be more influenced by environmental factors in ASD [16], see review [19]. These findings are informative regarding the neurobiological abnormalities that are associated with ASD, especially regarding the control for potential confounding sources of variability. However, these studies included relatively small sample sizes and did not compare $\mathrm{MZ}$ and $\mathrm{DZ}$ twins, which would help identify whether genetic or environmental factors are associated with the development of specific neurobiological differences.

In this investigation, we examine structural measures of the brain in MZ and DZ twin pairs with and without ASD to assess the influence of genetic and environmental factors on brain size. Based on previous investigations [11, 12, 16, 17], we hypothesized that cerebral tissue volume would be primarily influenced by genetic factors (i.e., MZ correlations would be significantly higher than DZ correlations) in both ASD and TD twins but that cerebellar volume, especially WM, would be influenced by environmental factors to a greater extent in ASD [16]. Surface area and cortical thickness are highly related to brain volume, but these components are genetically and phenotypically independent [13, 14] and exhibit different developmental trajectories [20, 21], suggesting that there may be differential contributions from genetic/ environmental influences in ASD. Based on the relatively high estimates of environmental influences on gyrification in TD twins [22] and the lack of concordance in MZ ASD twin pairs [18], we also predicted that curvature of the brain would be largely influenced by environmental factors in both ASD and TD twin pairs. Examining the relationship between genetic and environmental interactions on the previously reported brain size differences in ASD will help elucidate the possible etiological pathways from which these differences arise. This could help account for more of the neurobiological heterogeneity across individuals. Furthermore, identifying the neurobiological pathways that are affected by environmental factors in ASD will improve neurodevelopmental models, which will be an important step towards increasing the potential for neurobiological stratification in the future.

\section{Methods and materials}

\section{Participants}

Ninety same-sex twin pairs (male/female) aged 6-15 years, in which at least one twin was diagnosed with ASD or both were TD, were recruited to participate in this study. Participants with ASD were initially identified from the California Autism Twin Study [10] (27\%) and Interactive Autism Network Research Database (33\%). Additional twin pairs with ASD and TD control twin pairs were recruited from local/online advertisements. Exclusionary criteria included any evidence of genetic/metabolic disorders, history of traumatic head injury or asphyxia at birth, unstable medical conditions, or magnetic resonance imaging (MRI) contraindication. Control participants were also excluded if there was history of learning disabilities or severe affective/ psychiatric disorders (e.g., ASD, ADHD, schizophrenia, or major depression), as assessed with parent-report questionnaires, or a full-scale IQ (FSIQ) $<70$, as assessed with the Stanford-Binet Intelligence Scales, Fifth Edition [23]. The Child Behavioral Checklist (CBCL) [24] was also collected and all TD twins exhibited $T$-scores $\leq 70$ for Depressive, Anxiety, Somatic, Attention Deficit/Hyperactivity, Oppositional Defiant, Sluggish Cognitive Tempo, Obsessive Compulsive, and Stress Problems subscales, suggesting there were no clinically-relevant behavioral symptoms in our control group. For ASD twin pairs, clinical diagnosis was confirmed with the Autism Diagnostic Interview-Revised (ADI-R) [25] and Autism Diagnostic Observation Schedule, 2nd Edition (ADOS-2) [26], and participants were evaluated on an individual basis to determine his/her ability to complete study procedures. An initial power analysis that was based on 80 probands and 40 unrelated controls, allowing for $20 \%$ missing data, was completed with the STATA [27] program "powerlog" and indicated sufficient power to detect group-related differences within the expected effect size range.

Autism-related symptoms were compared between groups with the Social Responsiveness Scale (SRS) [28] 
and Short Sensory Profile (SSP) [29]. Handedness and socioeconomic status (SES) were assessed using the Edinburgh Handedness Inventory [30] and the Hollingshead method [31], respectively. Zygosity was confirmed from saliva samples based on nine short tandem repeat loci and the $\mathrm{X} / \mathrm{Y}$ amelogenin. Concordance on all markers was considered $\mathrm{MZ}$ whereas discordance for at least one marker was considered DZ [10]. The methodology of the study was approved by the Institutional Review Board, and written informed consent was obtained from parents and assent from participants. Additional information on the participants and general study design was reported previously $[32,33]$.

\section{MRI acquisition and processing}

MRI was conducted at two sites within the same institution, Lucile Packard Children's Hospital and the Richard M. Lucas Center for Imaging, on identical GE 3T MR750 scanners (Waukesha, Wisconsin, USA) using a standard 8-channel head coil. For individuals with ASD that were unable to remain motionless, sedation with propofol was administered under the supervision of an anesthesiologist at a rate of $200-300 \mathrm{mcg} / \mathrm{kg} /$ min to induce light procedural sedation. Two T1-weighted IR SPGR echo pulse sequence images were acquired from each participant (188 coronal slices, $\mathrm{TR}=8.15 \mathrm{~ms}, \mathrm{TE}=3.24 \mathrm{~ms}$, inversion time $=600 \mathrm{~ms}$, flip angle $=12$ degrees, slice thickness $=1.2 \mathrm{~mm}, \quad F O V=22 \times 22 \mathrm{~cm}$, in-plane resolution $=$ $0.86 \times 0.86$, and acquisition matrix size $=256 \times 192 \mathrm{~mm}$, $\mathrm{NEX}=1$ ), and the highest quality anatomical image was selected for further analysis.

Cortical reconstruction and volumetric segmentation was performed using FreeSurfer [34] (http://surfer.nmr.mgh.ha rvard.edu) and the Desikan-Killiany atlas [35]. Trained raters visually inspected all automated procedures and manually edited segmentations when errors were present. To evaluate potential site effects, two sets of twins (i.e., 4 participants) who did not require sedation were scanned at both locations. Repeated measures comparisons indicated an $\sim 6 \%$ difference in total brain volume between sites. Affected scans (ASD and TD) were transformed, prior to segmentation, using the FSL linear transformation package FLIRT [36] with standard sinc interpolation to FSL standard orientation images. The transformation matrix (Supplemental Table S1) was designed to minimize our site-specific differences in brain volume, surface area, and thickness across all parameters. Global brain measures included volume estimates of cortical and cerebellar GM and WM, subcortical GM, the brainstem and ventricular volume (lateral + inferior lateral $+3 \mathrm{rd}+4$ th ventricles). Total surface area and mean cortical thickness and curvature were also evaluated.

\section{Statistical analyses}

Intra-class correlations (ICC), controlling for variation associated with gender and diagnosis, were first generated in all $\mathrm{MZ}$ and $\mathrm{DZ}$ twin pairs to examine general twin pair differences in structural brain measures and ensure that our data met the basic assumptions for twin modeling. Analyses were performed with STATA [27] under the DeFries-Fulker model [37] framework, which is not constrained by the assumptions of the ACE model [38]. ICCs within ASD and TD twin pairs, excluding those discordant for ASD, were then examined separately with the same approach and were compared between zygosity groups with Fisher's z transformation to provide quantitative comparisons of twin pair differences between groups. Due to the sample size and basic analysis approach, discordant twin pairs were excluded from the diagnostic group specific analyses to remove the additional variability between twins with and without autism-related symptoms and increase generalizability to the greater ASD population. The ACE model for broad sense heritability was then calculated based on Falconer's formula [39] to estimate the contribution of genetic and environmental factors on variation of structural brain measures. The ACE model estimates the proportions of variation in a trait of interest (e.g., structural brain measures) that are related to additive genetic factors $\left(a^{2}\right)$ and common/ shared $\left(c^{2}\right)$ or unique $\left(e^{2}\right)$ environmental influences. Model components are generated by comparing trait variability in MZ versus DZ twin pairs, who share a common environment but differ in genetic influences by a known quantity (i.e., $50 \%$ ). ACE modeling was completed utilizing a bootstrapping method across 1000 repetitions. When A or C was non-significant, a simpler AE or CE model was utilized. Although diagnostic group comparisons were not the primary focus of the current investigation, comparisons between ASD and TD twins (Supplementary Table S2) and within twin pairs discordant for ASD (Supplementary Table S3) were included in the supplementary materials. In general, there were no major group differences in structural brain measures, especially after correction for multiple comparisons. These findings are not surprising because a relative "normalization" of global structural brain measures has been previously reported in individuals with ASD at similar developmental periods [15].

\section{Results}

\section{Participants}

T1-weighted images were acquired from 180 participants that comprised 90 twin pairs (55 ASD; 35 TD) (Table 1). 


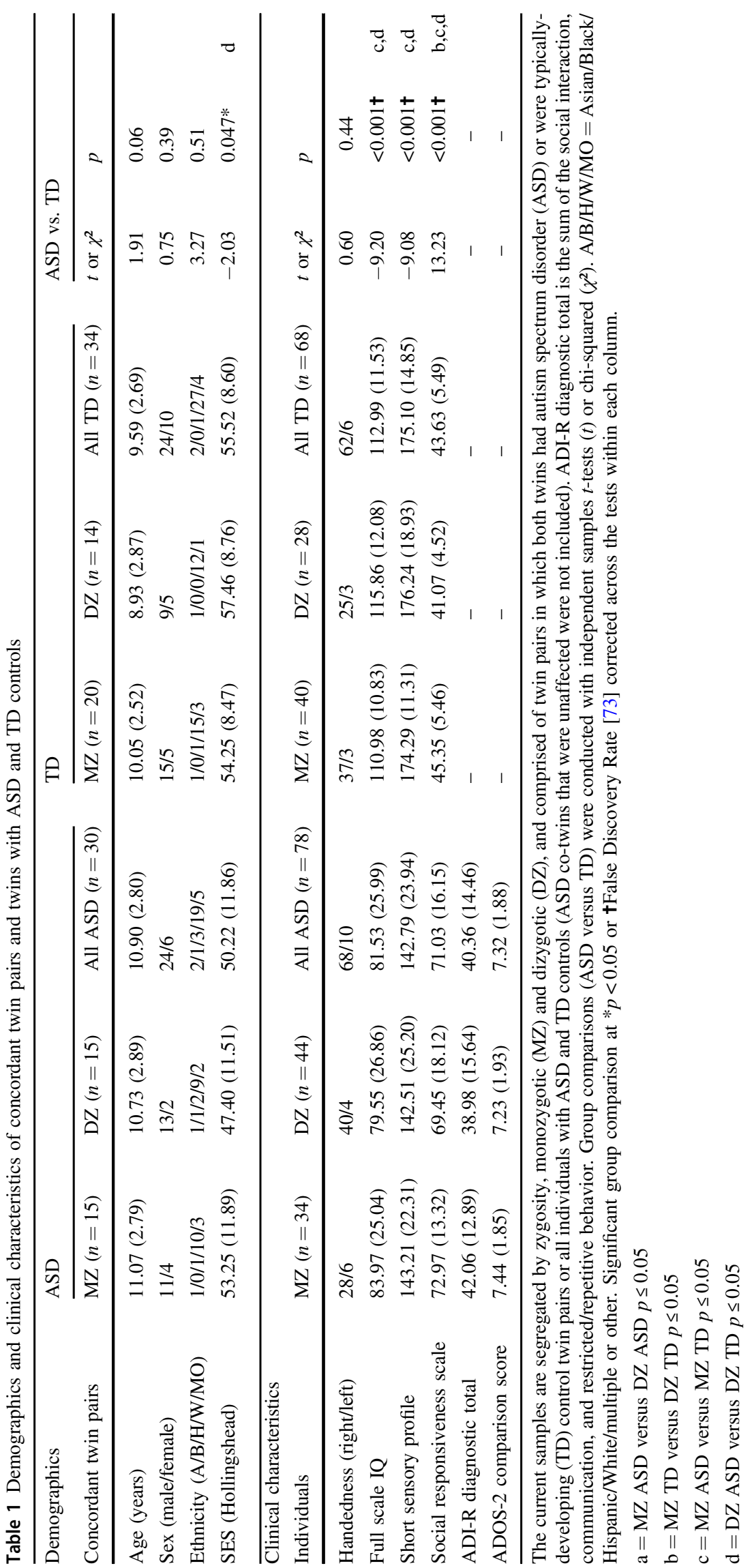




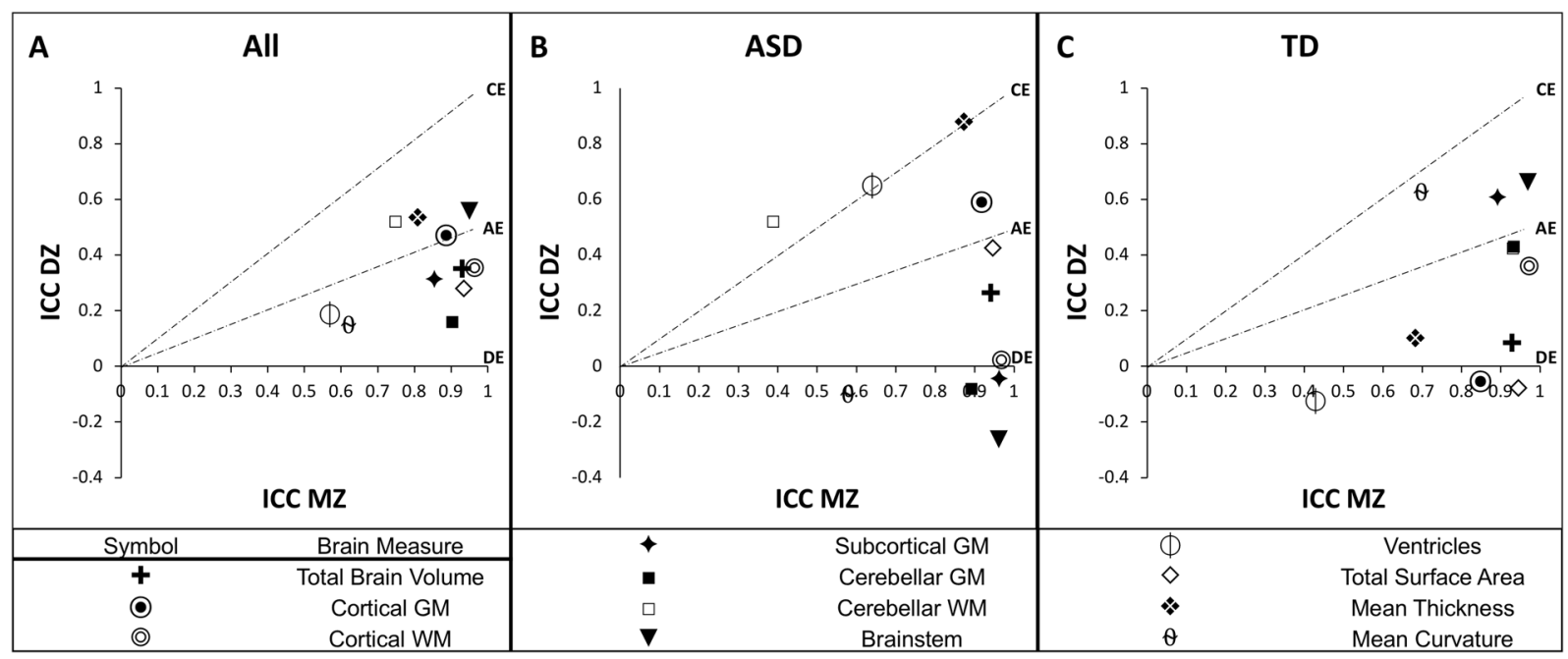

Fig. 1 ICCs in ACE model space. Intra-class correlation coefficients (ICC) in a all participants, adjusted for diagnosis and gender, and separately within twin pairs in which both twins were diagnosed with $\mathbf{b}$ autism spectrum disorder (ASD) or were $\mathbf{c}$ typically-developing (TD) controls, adjusted for gender, were generated within monozygotic (MZ)

Good quality scans were available from 30 twin pairs (15 MZ; 15 DZ) in which both twins had ASD, 18 twin pairs (4 MZ; 14 DZ) that were discordant for ASD, and 34 TD twin pairs (20 MZ; $14 \mathrm{DZ}$ ). Discordance for ASD was defined by one twin meeting diagnostic criteria on the ADI-R and ADOS whereas the other twin did not meet criteria for either ASD or the broader autism phenotype, in which subthreshold ASD-related impairments are indicated. There were no group differences in age, sex, ethnicity, or handedness, $p$ $<0.05$ in all instances. Although SES was slightly lower in the ASD group, $p=0.047$, primarily based on DZ twins, $p$ $=0.016$, there were no other zygosity by diagnostic group differences, $p>0.05$. Additionally, adjusting for SES did not significantly alter the reported ICCs or subsequent modeling; thus, unadjusted estimates are discussed in more detail.

As expected, twins with ASD exhibited more social deficits (based on the SRS), $p<0.001$, sensory-processing abnormalities, $p<0.001$, and lower FSIQ, $p<0.001$, compared to TD twins, with generally no zygosity group differences within the ASD and TD samples, $p>0.05$. The TD zygosity subgroups did exhibit a difference in total SRS scores, $p<0.001$; however, both subgroups $\left(\mathrm{SRS}_{\mathrm{MZ}}=\right.$ 45.35, $\mathrm{SD}=5.46 ; \mathrm{SRS}_{\mathrm{DZ}}=41.07, \mathrm{SD}=4.52$ ) were well below the threshold for clinically-relevant autism-related symptoms, which is between 65 and 70 depending on gender [40].

\section{Intra-class correlations (ICC) in $\mathrm{MZ}$ and $\mathrm{DZ}$ twin pairs}

Examining all twin pairs regardless of diagnosis, we found the anticipated pattern of ICC measures within and between zygosity subgroups, with ICCs generally positive, and dizygotic (DZ) twin pairs and are displayed in relation to ACE model space $[\mathrm{a}=$ additive genetics; $\mathrm{c}=$ shared family environment; $\mathrm{e}=$ unique environment; $d=$ genetic dominance]. Brain structures above or near the $\mathrm{CE}$ line are primarily environmentally-mediated whereas brain structures below or near the DE line are primarily genetically-mediated

significant, and higher in MZ compared to DZ twins (Fig. 1a; Supplementary Table S4). The only exceptions were found in DZ twins in which cerebellar GM, $p=0.23$, ventricular volume, $p=0.27$, and mean curvature, $p=0.42$, correlations were not statistically different than zero. However, previous studies have also reported relatively low magnitude correlations within DZ twin pairs for these brain measures [41] and the MZ twin pair ICCs were all still significantly higher, even after controlling for multiple comparisons. Thus, our data generally met the basic assumptions of twin modeling and all of aforementioned global brain measures were further examined in the ASD and TD subgroups.

Examining the ASD and TD twin pairs separately (Table 2; Fig. 1b, c) revealed salient ICC differences between diagnostic groups. All ICC estimates for MZ TD twins were statistically significant, $p<0.05$ in all instances, and large magnitude ( $\sim 0.70$ to 0.95$)$, with the exception of ventricular volume $\left(r_{\mathrm{MZ}}=0.43, p=0.024\right)$. All ICC estimates for MZ ASD twin pairs were also statistically significant, $p<0.05$, with large magnitude correlations $(\sim 0.60$ to 0.95$)$ except for cerebellar WM $\left(r_{\mathrm{MZ}}=0.39, p=0.022\right)$, which was lower in MZ ASD compared to MZ TD twins $\left(r_{\mathrm{MZ}}=0.93, \quad p<0.001\right), \quad p<0.001$. In contrast, ICCs for subcortical GM $\left(r_{\mathrm{MZ}}=0.96 ; r_{\mathrm{MZ}}=0.87 ; p=0.029\right)$ and mean thickness $\left(r_{\mathrm{MZ}}=0.89 ; r_{\mathrm{MZ}}=0.68 ; p=0.043\right)$ were higher in MZ ASD compared to MZ TD twins, respectively. However, these differences did not survive correction for multiple comparisons.

As expected, there was a much wider range of ICC estimates for both DZ TD ( -0.10 to 0.70$)$ and DZ ASD $(\sim-0.30$ to 0.90$)$ twins. Cortical GM $\left(r_{\mathrm{DZ}}=0.60\right.$; 


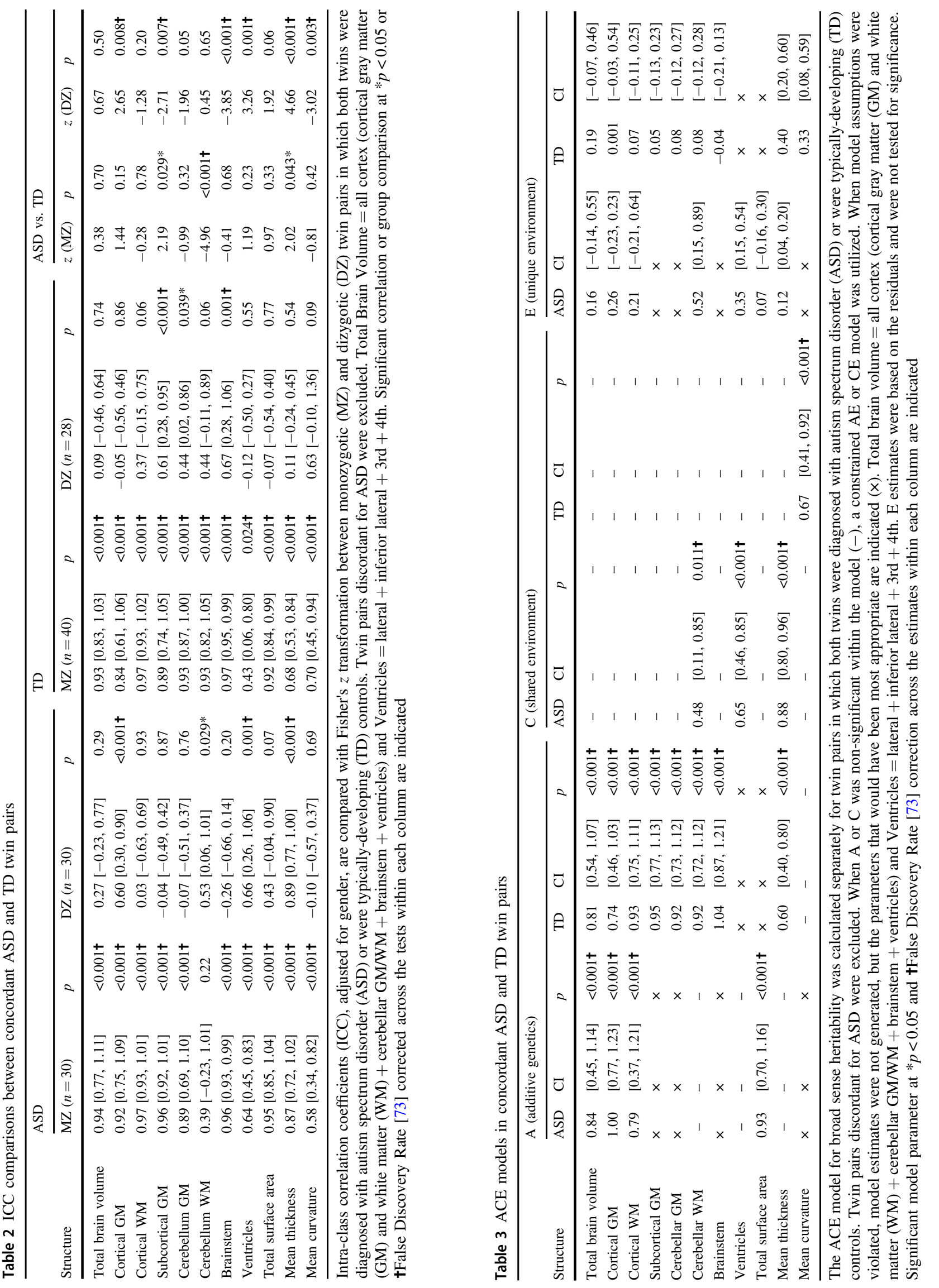


$\left.r_{\mathrm{DZ}}=-0.05 ; p=0.008\right)$, ventricular volume $\left(r_{\mathrm{DZ}}=0.66\right.$; $\left.r_{\mathrm{DZ}}=-0.12 ; p=0.001\right)$, and mean thickness $\left(r_{\mathrm{DZ}}=0.89\right.$; $\left.r_{\mathrm{DZ}}=0.11 ; p<0.001\right)$ showed higher ICCs in DZ ASD compared to DZ TD twin pairs, respectively. Additionally, ICCs for subcortical GM $\left(r_{\mathrm{DZ}}=-0.04 ; r_{\mathrm{DZ}}=0.61\right.$; $p=0.007), \quad$ the brainstem $\quad\left(r_{\mathrm{DZ}}=-0.26 ; \quad r_{\mathrm{DZ}}=0.67\right.$; $p<0.001)$, and mean curvature $\left(r_{\mathrm{DZ}}=-0.10 ; r_{\mathrm{DZ}}=0.63\right.$; $p=0.003$ ) were lower in DZ ASD compared to DZ TD twins.

\section{ACE modeling}

The main comparisons of interest were between MZ and DZ twin pairs within diagnostic groups (Table 3), which provides group-specific estimates for the influence of genetic versus environmental factors. Within TD twins, all global brain measures, except mean curvature, were best fit with the $\mathrm{AE}$ model. $a^{2}$ (additive genetic) estimates ranged from $\sim 0.6$ to 1 , suggesting that genetic influences primarily contributed to global brain measures in TD twins. Conversely, mean curvature was best fit with the $\mathrm{CE}$ model. The $c^{2}$ (common environment) estimate was 0.67 , suggesting that curvature was the only global structural measure that was primarily influenced by environmental factors in TD twins.

Within ASD twin pairs, there were significant deviations from the ACE models that were found in TD twin pairs. Similar to TD twins, the majority of global brain measures in ASD twins were best fit with the AE model. $a^{2}$ estimates ranged from $\sim 0.8$ to 1 . However, cerebellar WM, ventricular volume and mean thickness were best fit with the CE model. $c^{2}$ estimates ranged from $\sim 0.5$ to 0.9 . Thus, there appeared to be a greater influence of shared environmental factors on structural brain measures in twins with ASD compared to TD control twins.

There were a few global brain measures that could not be fit with the ACE models, such as ventricular volume and total surface area in TD twins and subcortical GM and mean curvature in ASD twins. The most probable reason for these fitting errors was the presence of negative correlations in the DZ subgroups, which caused violations of modeling assumptions. Based on the comparatively larger ICCs in the $\mathrm{MZ}$ twins, it appeared that these brain measures were also primarily genetically-mediated, including mean curvature in ASD twin pairs.

\section{Discussion}

In this investigation, brain size and other related global structural brain measures appeared to be primarily influenced by genetic factors in TD twins. The only exception was mean curvature, which was primarily associated with environmental factors. Similarly, genetic factors accounted for the majority of variation in brain size in twins with ASD, potentially to a larger extent for curvature and subcortical GM. However, there were also more environmental contributions to some brain structures in ASD. Cortical thickness and cerebellar WM volume were primarily influenced by environmental factors in ASD but not TD twin pairs. Cumulatively, these observations point to a possible increase in the vulnerability of certain brain structures to either genetic [3] or environmental [4] influences in individuals with ASD.

Findings from the present study are consistent with what has been previously reported in TD twin pairs [42], which indicated that brain size is primarily associated with genetic factors $(66-97 \%)$ [11, 12, 22, 43-48], including cortical GM (65-82\%) and WM (73-87\%) [11, 12, 45-48], surface area $(71-89 \%)[13,14]$ and cortical thickness $(69-81 \%)$ $[13,14,45]$. These estimates are similar to the current TD sample in which all brain measures that were assessed, excluding mean curvature, were also primarily influenced by genetic factors. Although some structural brain measures were not able to be evaluated with the ACE models, ICC comparisons between $\mathrm{MZ}$ and $\mathrm{DZ}$ twin pairs were in accordance with previous reports. Overall, these observations support the validity of our approaches and choice of control group.

Similar to the TD group, twins with ASD exhibited considerable genetic influences on global brain measures. Based on ICC comparisons between MZ and DZ twin pairs, total brain volume, cortical GM/WM, subcortical/cerebellar $\mathrm{GM}$, the brainstem, and surface area, appeared to be primarily genetically-mediated in ASD, which was supported by ACE modeling for total brain volume, cortical GM/WM and surface area. These findings suggest that the aforementioned reports of macrocephaly/early brain size differences in individuals with ASD [15] may have been associated with genetic factors, which is consistent with reports of an association between brain size and altered gene expression profiles in ASD [49]. Some brain measures may have also been more genetically-mediated in twins with ASD. We observed a higher additive genetic estimate for cortical GM in ASD compared to TD twin pairs, but this could have been associated with the non-significant DZ correlations. Similarly, there were several brain measures that exhibited extremely high MZ correlations and extremely low DZ correlations in ASD twins, suggesting a potentially larger impact of genetic influences. However, statistically significant ICC differences across diagnostic groups, which would support clinically-relevant alterations, were mostly absent and the assumptions of ACE modeling were not met. Importantly, the potential genetic dominance effect that was observed for subcortical GM in twins with ASD was supported by diagnostic group comparisons of ICC estimates, which indicated significantly higher ICCs in 
MZ ASD compared to MZ TD twins and significantly lower ICCs in DZ ASD compared to DZ TD twins. Although previous investigations of TD twin pairs have reported considerable genetic influences on subcortical GM [43, 45, 46], those estimates were much lower than what was observed in the current ASD sample. Subcortical GM structures have also been previously implicated in ASD, with reports of larger volumes of the caudate $[50,51]$ and globus pallidus/putamen $[52,53]$ but smaller volume of the thalamus [54, 55]. Thus, genetic influences on brain size appear to be similar in children and adolescents with and without ASD, at least in terms of magnitude, but there are some structures, such as subcortical GM, that may be more heavily genetically-influenced in individuals with ASD.

In contrast to the other structural brain measures that were primarily genetically-mediated, mean curvature, which is associated with gyrification of the brain [56], appears to be primarily environmentally-mediated in TD twins. This finding was supported by both ICC comparisons and ACE modeling and is consistent with previous investigations that indicated gyral patterns are primarily influenced by nongenetic factors during typical development [22]. Conversely, curvature of the brain appeared to be more genetically-mediated in twins with ASD, based on ICC comparisons, albeit the DZ ICC did not reach significance and could not be modeled with the ACE approach. The development of gyrification patterns is partially driven by WM connections within neuronal circuits [57]. These "connectivity" patterns are primarily established during the third trimester of fetal development but continue to mature during postnatal development via synaptic pruning and dendritic arborization [58]. Environmentally-mediated changes in gyrification could reflect adaptive experiencebased changes in neuronal connections. The finding of increased genetic influences on curvature in ASD may suggest that there is less adaptive experience-based alterations, which could be related to the reports of abnormal connectivity in ASD [59]. The only previous investigation of gyrification in twins with ASD reported ICCs that were lower ( -0.58 to 0.15$)$ [18] than the current investigation $(-0.10$ to 0.58$)$. However, that sample was primarily comprised of discordant twin pairs, which likely reduced the magnitude. These observations highlight the complexity of ASD in which some brain structures may be more influenced by genetic factors, while others may be more impacted by environmental factors.

Twins with ASD also exhibited increased environmental influences on some structural brain measures, such as cortical thickness, which was supported by both ICC comparisons and ACE modeling. Cortical thickness is primarily genetically-mediated during typical development [13, 14, 45] but appeared to be primarily environmentally-mediated in the current ASD sample. Cortical thickness has been previously implicated in ASD [60, 61] with evidence suggesting a relationship with the aforementioned early overgrowth and later normalization. Existing evidence also indicates that surface area and cortical thickness are largely genetically-independent [13, 14]. Based on ICC comparisons, the current findings expand on this line of research and suggest that whereas surface area is primarily genetically-mediated in both TD and ASD twin pairs, cortical thickness may be influenced by environmental factors to a larger extent in ASD. The effects of environmental factors on altered developmental trajectories of cerebral size in children with ASD may exert their influence via pathways that modulate cortical thickness.

Additional findings from the current investigation also indicate the possible existence of increased environmental influences on cerebellar WM and ventricular volume in ASD. Previous estimates of genetic influences on cerebellar $(49-88 \%)[12,44,46-48,62]$ and ventricular (0-92\%) [11, 12, 44, 46, 47, 63-65] volume in TD twins have been variable across investigations. The extreme variability regarding ventricular volume makes interpretation of potential ASD-related differences difficult, but estimates of genetic influences on cerebellar WM are still relatively high across studies. The magnitude of environmental influences on cerebellar WM in the current ASD sample are strikingly different than the current and previous estimates for TD twins for both ICC comparisons and ACE modeling estimates. This finding is also supported by the only previous investigation of cerebellar WM in twins with ASD [16], in which MZ twins concordant for ASD exhibited significantly higher ICCs compared to MZ discordant twin pairs. Cerebellar WM has also been previously implicated in ASD $[66,67]$. Although these alterations are somewhat variable across investigations, prenatal loss of Purkinje cells in the cerebellum, which contribute axons to cerebellum WM, is consistently reported [68]. The cerebellum may also be particularly sensitive to the effects of environmental stressors during prenatal development $[11,69,70]$, suggesting that environmental factors affecting the in utero environment may be associated with the development of volumetric abnormalities in this structure.

There are several limitations of the current investigation that should be considered. Our sample is rather large for an MRI study but was not large enough at times to apply more advanced twin modeling techniques, such as Defries-Fulker regression [37, 38]. This was especially evident in the DZ twin pairs in which non-significant and/or negative ICCs were found for several measures. The examination of twin pairs, which include related individuals, may have also affected the variability in our general diagnostic group comparisons. However, comparisons of only one twin from each pair were generally in accordance with the reported findings. The basic ACE model that was utilized assumes 
that shared environmental effects are the same for MZ and DZ twin pairs, which may allocate some environmental influences to the genetic factor, and also assumes there are no gene by environment interactions, which cumulatively may cause an overestimation of genetic influences. The concordance rates for ASD in the current sample were also somewhat higher compared to earlier estimates [7], especially for DZ twins, but were similar to recent findings from a more diverse population sample that was assessed with updated diagnostic criteria [10]. The two separate scanners that were utilized could have also introduced additional variability, so transformation of approximately one-third of the neuroimaging data was applied. Analyses excluding these data did not significantly differ from those reported for the full dataset. Finally, our findings indicate the magnitude with which genetic and environmental factors may influence brain size, not the specific factors that are driving these effects, and the global level measures provide information on the overall components (or compartments) that may play a role in the brain pathology of ASD but not the local contributing factors $[46,48,71,72]$.

In summary, brain size is primarily genetically-mediated during typical development, and our preliminary findings indicate a similar observation for individuals with ASD. However, genetic factors may influence subcortical GM to a larger extent in ASD, and environmental factors may exert a greater impact on the development of some brain structures, such as cortical thickness and cerebellar WM. Additional observations also indicated that individuals with ASD may undergo less adaptive environmentally-mediated changes in curvature/gyrification. Future investigations should assess larger twin samples to replicate these findings and additional efforts should be made to include more DZ twin pairs. Younger twin samples should also be evaluated to identify the genetic and environmental factors that influence brain structure during early development in ASD.

Acknowledgements We would like to thank Sean Berquist, Serena Tamura, and Phoebe Cates for their data acquisition support and the many behavioral technicians that conducted the cognitive/behavioral testing. We would like to especially thank the participants and their families, many of whom traveled great distances. This work was supported by an award from the National Institute of Mental Health (R01MH083972 to A.Y.H.). J.P.H was supported by the Bass Society Pediatric Fellowship Program.

\section{Compliance with ethical standards}

Conflict of interest The authors declare that they have no conflict of interest.

Publisher's note: Springer Nature remains neutral with regard to jurisdictional claims in published maps and institutional affiliations.

Open Access This article is licensed under a Creative Commons Attribution 4.0 International License, which permits use, sharing, adaptation, distribution and reproduction in any medium or format, as long as you give appropriate credit to the original author(s) and the source, provide a link to the Creative Commons license, and indicate if changes were made. The images or other third party material in this article are included in the article's Creative Commons license, unless indicated otherwise in a credit line to the material. If material is not included in the article's Creative Commons license and your intended use is not permitted by statutory regulation or exceeds the permitted use, you will need to obtain permission directly from the copyright holder. To view a copy of this license, visit http://creativecommons. org/licenses/by/4.0/.

\section{References}

1. Baio J, Wiggins L, Christensen DL, Maenner MJ, Daniels J, Warren Z, et al. Prevalence of autism spectrum disorder among children aged 8 years-autism and developmental disabilities monitoring network, 11 Sites, United States, 2014. MMWR Surveill Summ. 2018;67:1-23.

2. APA. Diagnostic and statistical manual of mental disorders: DSMV: Arlington, VA, 2013.

3. Miles JH. Autism spectrum disorders-a genetics review. Genet Med. 2011;13:278.

4. Modabbernia A, Velthorst E, Reichenberg A. Environmental risk factors for autism: an evidence-based review of systematic reviews and meta-analyses. Mol Autism. 2017;8:13.

5. Geschwind DH, Levitt P. Autism spectrum disorders: developmental disconnection syndromes. Curr Opin Neurobiol. 2007;17:103-11.

6. Amaral DG, Schumann CM, Nordahl CW. Neuroanatomy of autism. Trends Neurosci. 2008;31:137-45.

7. Bailey A, Le Couteur A, Gottesman I, Bolton P, Simonoff E, Yuzda E, et al. Autism as a strongly genetic disorder: evidence from a British twin study. Psychol Med. 2009;25:63.

8. Folstein SE, Rutter M. Infantile autism: a genetic study of 21 twin pairs. J Child Psychol Psychiatry. 2006;18:297-321.

9. Steffenburg S, Gillberg C, Kellgren L, Andersson L, Gillberg IC, Jakobsson G, et al. A twin study of autism in Denmark, Finland, Iceland, Norway and Sweden. J Child Psychol Psychiatry. 2006;30:405-16.

10. Hallmayer J, Cleveland S, Torres A, Phillips J, Cohen B, Torigoe T, et al. Genetic heritability and shared environmental factors among twin pairs with autism. JAMA Psychiatry. 2011;68:1095102.

11. Baare WF, Hulshoff Pol HE, Boomsma DI, Posthuma D, de Geus EJ, Schnack HG, et al. Quantitative genetic modeling of variation in human brain morphology. Cereb Cortex. 2001;11:816-24.

12. Wallace GL, Schmitt JE, Lenroot R, Viding E, Ordaz S, Rosenthal MA, et al. A pediatric twin study of brain morphometry. J Child Psychol Psychiatry. 2006;47:987-93.

13. Panizzon MS, Fennema-Notestine C, Eyler LT, Jernigan TL, Prom-Wormley E, Neale M, et al. Distinct genetic influences on cortical surface area and cortical thickness. Cereb Cortex. 2009;19:2728-35.

14. Winkler AM, Kochunov P, Blangero J, Almasy L, Zilles K, Fox PT, et al. Cortical thickness or grey matter volume? The importance of selecting the phenotype for imaging genetics studies. Neuroimage. 2010;53:1135-46.

15. Courchesne E, Campbell K, Solso S. Brain growth across the life span in autism: age-specific changes in anatomical pathology. Brain Res. 2011;1380:138-45.

16. Kates WR, Burnette CP, Eliez S, Strunge LA, Kaplan D, Landa R, et al. Neuroanatomic variation in monozygotic twin pairs discordant for the narrow phenotype for autism. Am J Psychiatry. 2004;161:539-46. 
17. Mitchell SR, Reiss AL, Tatusko DH, Ikuta I, Kazmerski DB, Botti $\mathrm{J}$-AC et al. Neuroanatomic alterations and social and communication deficits in monozygotic twins discordant for autism disorder. Am. J. Psychiatry. 2009;166:917-25.

18. Kates WR, Ikuta I, Burnette CP. Gyrification patterns in monozygotic twin pairs varying in discordance for autism. Autism Res. 2009;2:267-78.

19. Mevel K, Fransson P, Bölte S. Multimodal brain imaging in autism spectrum disorder and the promise of twin research. Autism. 2015;19:527-41.

20. Wierenga LM, Langen M, Oranje B, Durston S. Unique developmental trajectories of cortical thickness and surface area. Neuroimage. 2014;87:120-6.

21. Ecker $\mathrm{C}$, Ginestet $\mathrm{C}$, Feng $\mathrm{Y}$, et al. Brain surface anatomy in adults with autism: the relationship between surface area, cortical thickness, and autistic symptoms. JAMA Psychiatry. 2013;70:59-70.

22. Bartley AJ, Jones DW, Weinberger DR. Genetic variability of human brain size and cortical gyral patterns. Brain. 1997;120:257-69.

23. Roid GH. Stanford-Binet intelligence scales. Itasca, IL: Riverside Publishing; 2003

24. Achenbach TM. The Child Behavior Checklist and related instruments. The use of psychological testing for treatment planning and outcomes assessment, 2nd ed. Mahwah, NJ: Lawrence Erlbaum Associates Publishers; 1999, p. 429-66.

25. Lord C, Rutter M, Le Couteur A. Autism diagnostic interviewrevised: a revised version of a diagnostic interview for caregivers of individuals with possible pervasive developmental disorders. J Autism Dev Disord. 1994;24:659-85.

26. Lord C, Rutter M, DiLavore P, Risi S, Gotham K, Bishop S. Autism diagnostic observation schedule (ADOS-2). 2nd ed. Torrance, CA: Western Psychological Corporation; 2012.

27. StataCorp. Stata Statistical Software. vol. Release 15. College Station, TX: StateCorp LLC; 2017.

28. Constantino JN, Gruber CP. Social responsiveness scale (SRS). Los Angeles, CA: Western Psychological Services; 2007.

29. Dunn W. Short sensory profile. San Antonio, TX: Psychological Corporation; 1999.

30. Oldfield RC. The assessment and analysis of handedness: the Edinburgh inventory. Neuropsychologia. 1971;9:97-113.

31. Hollingshead AB Four factor index of social status. Unpublished manuscript edn. New Haven, CT: Yale University; 1975.

32. Hegarty JP, Gu M, Spielman DM, Cleveland SC, Hallmayer JF, Lazzeroni LC, et al. A proton MR spectroscopy study of the thalamus in twins with autism spectrum disorder. Prog Neuropsychopharmacol Biol Psychiatry. 2018;81:153-60.

33. Monterrey JC, Philips J, Cleveland S, Tanaka S, Barnes P, Hallmayer JF, et al. Incidental brain MRI findings in an autism twin study. Autism Res. 2017;10:113-20.

34. Fischl B. FreeSurfer. Neuroimage. 2012;62:774-81.

35. Desikan RS, Ségonne F, Fischl B, Quinn BT, Dickerson BC, Blacker D, et al. An automated labeling system for subdividing the human cerebral cortex on MRI scans into gyral based regions of interest. Neuroimage. 2006;31:968-80.

36. Jenkinson $M$, Bannister $P$, Brady $M$, Smith S. Improved optimization for the robust and accurate linear registration and motion correction of brain images. Neuroimage. 2002;17:825-41.

37. DeFries JC, Fulker DW. Multiple regression analysis of twin data. Behav Genet. 1985;15:467-73.

38. Lazzeroni LC, Ray A. A generalized Defries-Fulker regression framework for the analysis of twin data. Behav Genet. 2013;43:85-96.

39. Falconer DS. Introduction to quantitative genetics, 2nd ed. London: Longman; 1981.

40. Constantino JN, Gruber CP. The social responsiveness scale. Los Angeles: Western Psychological Services; 2002.
41. Kremen WS, Prom-Wormley E, Panizzon MS, Eyler LT, Fischl B, Neale MC, et al. Genetic and environmental influences on the size of specific brain regions in midlife: The VETSA MRI study. Neuroimage. 2010;49:1213-23.

42. Peper JS, Brouwer RM, Boomsma DI, Kahn RS, Hulshoff Pol HE. Genetic influences on human brain structure: a review of brain imaging studies in twins. Hum Brain Mapp. 2007;28:464-73.

43. Pennington BF, Filipek PA, Lefly D, Chhabildas N. A twin MRI study of size variations in the human brain. J Cogn Neurosci. 2000;12:223-32.

44. Wright IC, Sham P, Murray RM, Weinberger DR, Bullmore ET. Genetic contributions to regional variability in human brain structure: methods and preliminary results. Neuroimage. 2002;17:256-71.

45. Yoon U, Fahim C, Perusse D, Evans AC. Lateralized genetic and environmental influences on human brain morphology of 8-yearold twins. Neuroimage. 2010;53:1117-25.

46. Blokland GAM, de Zubicaray GI, McMahon KL, Wright MJ. Genetic and environmental influences on neuroimaging phenotypes: a meta-analytical perspective on twin imaging studies. Twin Res Hum Genet. 2012;15:351-71.

47. Peper Jiska S, Schnack Hugo G, Brouwer Rachel M, Van Baal GCM, Pjetri E, Székely E, et al. Heritability of regional and global brain structure at the onset of puberty: a magnetic resonance imaging study in 9-year-old twin pairs. Hum Brain Mapp. 2009;30:2184-96.

48. Yoon U, Perusse D, Lee J-M, Evans AC. Genetic and environmental influences on structural variability of the brain in pediatric twin: deformation based morphometry. Neurosci Lett. 2011;493:8-13.

49. Chow ML, Pramparo T, Winn ME, Barnes CC, Li H-R, Weiss L, et al. Age-dependent brain gene expression and copy number anomalies in autism suggest distinct pathological processes at young versus mature ages. PLoS Genet. 2012;8:e1002592.

50. Sears LL, Vest C, Mohamed S, Bailey J, Ranson BJ, Piven J. An MRI study of the basal ganglia in autism. Prog Neuropsychopharmacol Biol Psychiatry. 1999;23:613-24.

51. Hollander E, Anagnostou E, Chaplin W, Esposito K, Haznedar MM, Licalzi E, et al. Striatal volume on magnetic resonance imaging and repetitive behaviors in autism. Biol Psychiatry. 2005;58:226-32.

52. Herbert MR, Ziegler DA, Deutsh CK, O'Brien LM, Lange N, Bakardjiev A, et al. Dissociations of cerebral cortex, subcortical and cerebral white matter volumes in autistic boys. Brain. 2003;126:1182-92.

53. Sato W, Kubota Y, Kochiyama T, Uono S, Yoshimura S, Sawada R, et al. Increased putamen volume in adults with autism spectrum disorder. Front Hum Neurosci. 2014;8:957.

54. Tamura R, Kitamura $H$, Endo $T$, Hasegawa $N$, Someya $T$. Reduced thalamic volume observed across different subgroups of autism spectrum disorders. Psychiatry Res. 2010;184:186-8.

55. Tsatsanis KD, Rourke BP, Klin A, Volkmar FR, Cicchetti D, Schultz RT. Reduced thalamic volume in high-functioning individuals with autism. Biol Psychiatry. 2003;53:121-9.

56. Luders E, Thompson PM, Narr KL, Toga AW, Jancke L, Gaser C. A curvature-based approach to estimate local gyrification on the cortical surface. Neuroimage. 2006;29:1224-30.

57. Van Essen DC. A tension-based theory of morphogenesis and compact wiring in the central nervous system. Nature. 1997;385:313-8.

58. White T, Su S, Schmidt M, Kao C-Y, Sapiro G. The development of gyrification in childhood and adolescence. Brain Cogn. 2010;72:36-45.

59. Belmonte MK, Allen G, Beckel-Mitchener A, Boulanger LM, Carper RA, Webb SJ. Autism and abnormal development of brain connectivity. J Neurosci. 2004;24:9228. 
60. Hardan AY, Muddasani S, Vemulapalli M, Keshavan MS, Minshew NJ. An MRI study of increased cortical thickness in autism. Am J Psychiatry. 2006;163:1290-2.

61. Hardan AY, Libove RA, Keshavan MS, Melhem NM, Minshew NJ. A preliminary longitudinal magnetic resonance imaging study of brain volume and cortical thickness in autism. Biol Psychiatry; 66: 320-6.

62. Posthuma D, de Geus EJC, Neale MC, Hulshoff Pol HE, Baaré WEC, Kahn RS, et al. Multivariate genetic analysis of brain structure in an extended twin design. Behav Genet. 2000;30: 311-9.

63. Pfefferbaum A, Sullivan EV, Carmelli D. Morphological changes in aging brain structures are differentially affected by time-linked environmental influences despite strong genetic stability. Neurobiol Aging. 2004;25:175-83.

64. Pfefferbaum A, Sullivan EV, Swan GE, Carmelli D. Brain structure in men remains highly heritable in the seventh and eighth decades of life. Neurobiol Aging. 2000;21:63-74.

65. Reveley AM, Reveley MA, Chitkara B, Clifford C. The genetic basis of cerebral ventricular volume. Psychiatry Res. 1984;13:261-6.

66. Murakami JW, Courchesne E, Press GA, Yeung-Courchesne R, Hesselink JR. Reduced cerebellar hemisphere size and its relationship to vermal hypoplasia in autism. Arch Neurol. 1989;46:689-94.

67. Courchesne E, Karns CM, Davis HR, Ziccardi R, Carper RA, Tigue $\mathrm{ZD}$, et al. Unusual brain growth patterns in early life in patients with autistic disorder: an MRI study. Neurology. 2001;57:245-54.

68. Fatemi SH, Aldinger KA, Ashwood P, Bauman ML, Blaha CD, Blatt GJ, et al. Consensus paper: pathological role of the cerebellum in autism. Cerebellum. 2012;11:777-807.

69. Beversdorf DQ, Manning SE, Hillier A, Anderson SL, Nordgren RE, Walters SE, et al. Timing of prenatal stressors and autism. J Autism Dev Disord. 2005;35:471-8.

70. Sajdel-Sulkowska EM, Xu M, McGinnis W, Koibuchi N. Brain region-specific changes in oxidative stress and neurotrophin levels in autism spectrum disorders (ASD). Cerebellum. 2011;10:43-48.

71. Joshi AA, Lepore N, Joshi SH, Lee AD, Barysheva M, Stein JL, et al. The contribution of genes to cortical thickness and volume. Neuroreport. 2011;22:101-5.

72. Rimol LM, Panizzon MS, Fennema-Notestine C, Eyler LT, Fischl B, Franz CE, et al. Cortical thickness Is influenced by regionally specific genetic factors. Biol Psychiatry. 2010;67:493-9.

73. Benjamini Y, Hochberg Y. Controlling the false discovery rate: a practical and powerful approach to multiple testing. J R Stat Soc. 1995;57:289-300. 